\title{
Evaluation of Deterioration Damage for Liquid Oxygen/Hydrogen Combustion Chamber of Copper Alloy by Replica Method for Oxide Film*
}

\author{
Mitsuharu Shiwa ${ }^{1,4}$, Masao Hayakawa ${ }^{1}$, Tomoyuki Hashimoto ${ }^{2}$, Masao Takegoshi ${ }^{2}$ and Eiichi Sato ${ }^{3}$ \\ ${ }^{1}$ Research Center for Structural Materials, National Institute for Materials Science, Tsukuba 305-0047, Japan \\ ${ }^{2}$ Research and Development Directorate, Japan Aerospace Exploration Agency, Tsukuba 305-8505, Japan \\ ${ }^{3}$ Institute for Space and Astronautical Science, Japan Aerospace Exploration Agency, Sagamihara 252-0222, Japan \\ ${ }^{4}$ Faculty of Future Industry, Happy Science University, Chosei-gun, Chiba 299-4325, Japan
}

Non-destructive damage evaluation of rocket engine combustion chambers was carried out using a replica method that employs a rapidsetting silicone rubber molding agent. Scratch marks, grinding marks and the oxide film on the combustion side surface that developed after repeated burning tests were successfully transferred to a silicone rubber sheet, eliminating any need for complex surface treatment before examination. The sampled replicas showed traces of oxide film formation and the disappearance of the grinding marks and crack formations on the oxide films caused by to redox reactions during repeated use. The surface roughness of the light and dark lines parallel to the cooling channels and cracked oxide film was evaluated by laser scanning microscopy. We found the surface roughness parameter to be potentially useful for evaluating the level of damage sustained by reusable sounding rocket engines. [doi:10.2320/matertrans.MT-M2019386]

(Received December 25, 2019; Accepted June 5, 2020; Published August 25, 2020)

Keywords: damage evaluation, copper-alloy, combustion chamber, replica method, oxide film

\section{Introduction}

The mixture of liquid oxygen and liquid hydrogen used as propellant in rockets burns at up to $3000 \mathrm{~K}$. The inner wall of the combustion chamber must therefore be protected by cooling with liquid hydrogen $(20 \mathrm{~K})$, which is passed through channels of rectangular cross section in the wall of the combustion chamber. Owing to their good mechanical and heat conduction characteristics, $\mathrm{Cu}-\mathrm{Ag}-\mathrm{Zr}$ and $\mathrm{Cu}-\mathrm{Cr}-\mathrm{Zn}$ alloys are used as combustion chamber materials. $\mathrm{Cu}-\mathrm{Ag}-\mathrm{Zr}$ alloy was used in the Space Shuttle main engine, which was a repeatedly reusable rocket engine. The coolant and the combustion gas are separated by a 'cooling channel wall' of about $1 \mathrm{~mm}$ thick. Creep fatigue damage occurs inside the wall owing to the steep heat gradient and thermal strain it experiences. ${ }^{1)}$ The inner surface of the combustion chamber wall is subject to damage caused by the combustion gas atmosphere, which subjects the oxide layer formed on the surface to repeated oxidation and reduction, causing the formation of many micropores, which increase the surface roughness. Blanching and microcracks then occur at the grain boundaries in the copper alloy. ${ }^{2}$

The Japan Aerospace Exploration Agency (JAXA) is engaged in a project to develop a 100-times reusable engine for sounding rockets in which the combustion chamber is made of $\mathrm{Cu}-\mathrm{Cr}-\mathrm{Zn}$ alloy. ${ }^{3,4)}$ Figure 1 shows the engine's experimental combustion chamber and its cross-sectional structure. ${ }^{5)}$ From non-destructive inspection of the combustion chamber following cumulative starts and stops equivalent to 117 flight operations, we concluded that high-energy x-ray CT and ultrasonic thickness measurements could be used to measure the internal deformation of the chamber and the thickness of the cooling channel wall. ${ }^{5)} \mathrm{We}$ evaluated the eccentricity, surface irregularities, and thinning

*This Paper was Originally Published in Japanese in J. Japan Inst. Met. Mater. 83 (2019) 176-180. Equation (2) and the captions of Figs. 1, 3, 4, 6, 7 were slightly changed.

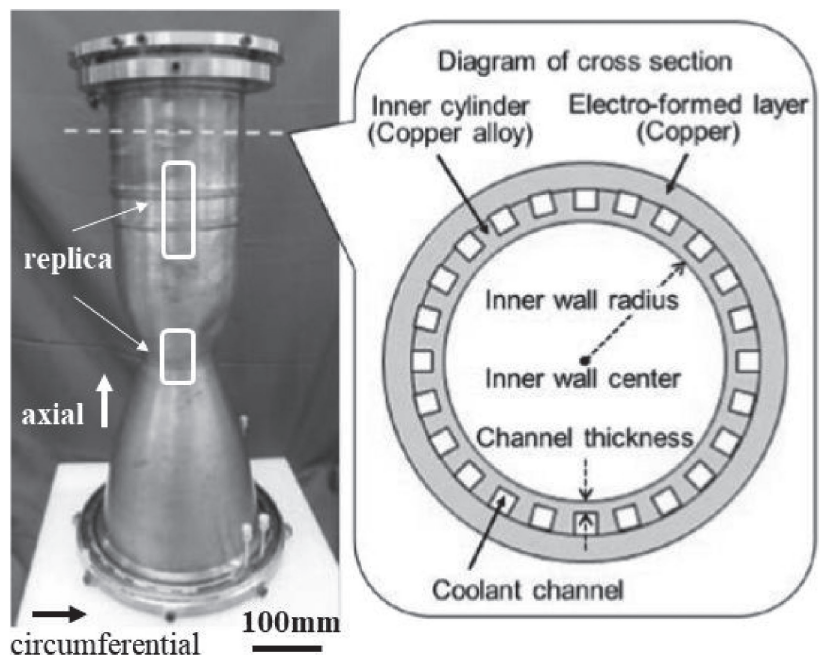

Fig. 1 Overview and cross section of the regeneratively-cooled combustion chamber.

of the wall and detected a difference in radius of $0.8 \mathrm{~mm}$ on the inner surface and uneven deformation upstream of the throat, which is the location of the maximum heat flux and where the inner wall temperature is the highest. ${ }^{5)}$

When evaluating deterioration, it is necessary to identify the mechanism of damage before deformation and cracks are generated as a result of the combustion chamber's operation, and to evaluate the degree of damage caused during their generation. We have been developing non-destructive testing methods for rockets with copper alloy combustion chambers and methods of evaluating their remaining life. ${ }^{6,7)}$

There are multiple methods of predicting the life expectancy of high-temperature equipment. Prediction of the residual life of repeatedly used high-temperature equipment, such as that used in power generation plants and chemical plants, is typically carried out using the diagnosis of boiler tubes. ${ }^{8,9)}$ However, for welded joints, the hardness measurement method and replica method, which are based on 
materials testing protocols, are most commonly used for surface inspections. Volume inspection uses nondestructive ultrasonic, radiographic and electromagnetic techniques.

The replica method involves polishing and etching metal surfaces, transferring the microstructure to acetate tape, and then observing it through a microscope. ${ }^{10)}$ Using this technique, the precipitation structure (the $\gamma$ phase) of IN738LC has been observed in the Ni-base superalloy used in gas turbine blades. ${ }^{11)}$ Surface cracks that developed during low-cycle fatigue testing of 316 stainless steel at room temperature and pressure have also been used to evaluate crack nucleation and propagation. ${ }^{12)}$ A replica method using rapid-curing silicone rubber has recently been developed that is able to transfer the characteristics of both the macroscopic surface shape and its microstructures. ${ }^{13)}$

Here we assessed the cooling channel wall of the combustion chamber of a reusable sounding rocket, which has a thickness of about $1 \mathrm{~mm}$. It is necessary to detect the initiation and propagation of micro surface cracks caused by the blanching phenomenon, in which the surface roughness increases with repeated oxidation and reduction of the oxide layer. $^{14,15)}$

When the replica method is applied to the combustion chamber of a reusable rocket, any reduction in the thickness of the cooling channel wall due to surface polishing must be avoided. Although evaluation of the blanching phenomenon on the surface might be more important than that of the oxidized surface state after combustion, we tested a method of inspecting the oxide film that forms on the surface.

\section{Experimental Procedure}

The combustion chamber used in the experiment is shown in Fig. 1. The inner cylinder, made of a $\mathrm{Cu}-\mathrm{Cr}-\mathrm{Zr}$ alloy, is $600 \mathrm{~mm}$ long, with an inner diameter of 170 to $270 \mathrm{~mm}$, and a throat portion of $100 \mathrm{~mm}$ wide.

The cooling channels are arranged at a pitch of about $1.5 \mathrm{~mm}$ in the axial direction of the inner cylinder. The test chamber burned liquid oxygen and liquid hydrogen for $3785 \mathrm{~s}$ in total, with 142 starts and stops (multiple ignition tests $^{4)}$ ) and thrust changes.

A two-part, rapid-set, silicone rubber molding agent (F5; pot life $5 \mathrm{~min}$, curing time $18 \mathrm{~min}$; Struers, Willich, Germany) was used for replica production. The components in the dispensing cartridge are mixed in the nozzle, extruded from the tip, and cured on the surface of the specimen. Since the dispensing gun is bulky, the tip of the mixing nozzle could not be brought close to the surface of the narrow part of the combustion chamber, so we attached a 20-cm-long silicone tube to the tip of the nozzle and applied the mixture by moving the tip of the tube. Because the cooling channel wall is very thin, surface treatment consisted only of cleaning it with a gauze cloth wetted with acetone; the surface was not mechanically polished or etched. Molds were cast at the nozzle throat, which is a high-stress part, and the parallel part of the inner wall (explained in Fig. 4), where oxide film remained on the surface (Fig. 1). The silicone replicas were observed under a stereomicroscope and a video microscope (VHX1000, Keyence, Osaka, Japan), and features of deformation and cracks were measured by a laser scanning microscope (LSM; VHK9710, Keyence) at a high scanning pitch of $2.8 \mu \mathrm{m}$.

\section{Experimental Results}

\subsection{Transcription capability of replica sheets}

After 142 combustion cycle tests, the surface of the inner cylinder of the combustion chamber produced less oxide film than might be expected, owing to the creation of a reducing atmosphere at the end of each combustion cycle. The entire surface therefore had a metallic luster close to that of new material, but with a black oxide film remaining in some parts.

Figure 2 shows a replica sheet taken from the parallel part of the inner cylinder. The replica comprised a single sheet measuring $40 \mathrm{~mm}$ wide and $180 \mathrm{~mm}$ long. The arrows in Figs. 2 to 6 indicate the circumferential direction of the combustion chamber's inner cylinder. The molding agent flowed almost uniformly onto the surface, allowing the curvature and surface conditions to be accurately transferred.

\subsection{Observation results of residual oxide film parts}

Figure 3 shows different surface roughness features in the parallel part: a circular smooth surface with a step in the center, and an uneven surface around it. Detailed observations suggest that crack-like scratches parallel to the axial direction were made during machining.

Figure 4 shows enlargements of the area around the step in Fig. 3. In the smooth surface at the center, scratch marks and

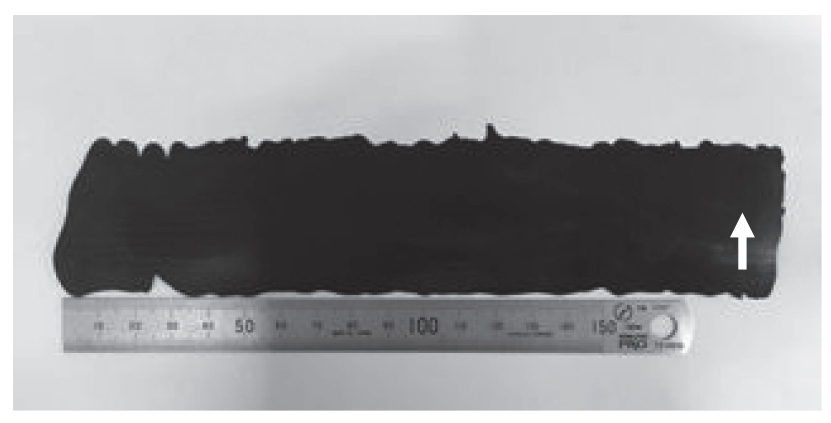

Fig. 2 Maximum size of replica sheets sampled at the parallel part of the inner wall.

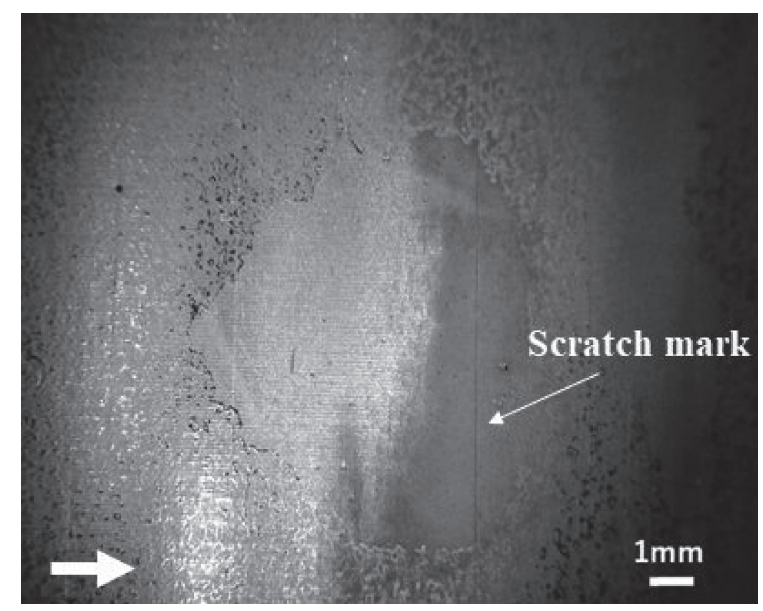

Fig. 3 Macroscopic images on the replica sheet of areas with different surface roughness on the parallel part of the inner wall. 


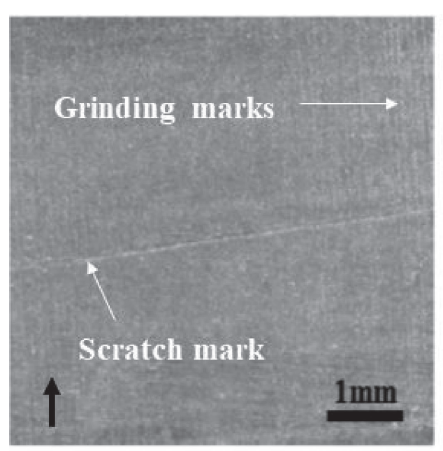

(a)

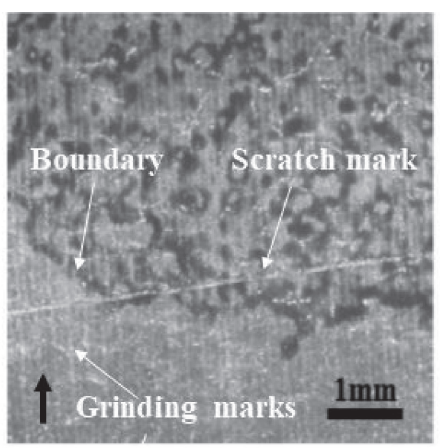

(b)

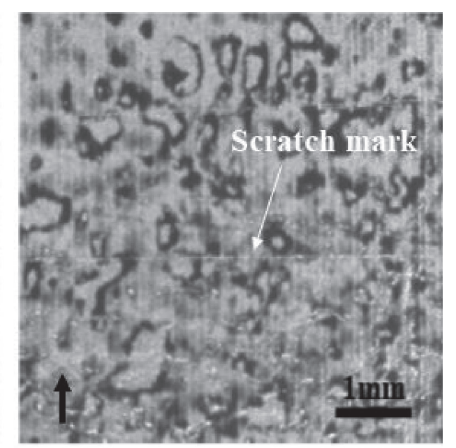

(c)

Fig. 4 Microscopic images on the replica sheet of regions with different surface roughness on the parallel part of the inner wall. (a) Smooth surface region, (b) boundary region between the smooth surface region and the rough surface region, (c) rough surface region.

regular grinding marks like vertical stripes parallel to the circumferential direction are clear (Fig. 4(a)). The boundary between the smooth surface and the uneven surface is clear (Fig. 4(b)). On the uneven surface, the oxidation products on the grains can be clearly seen with traces of grinding (Fig. 4(c)). The inner surface of the chamber corresponding to Fig. 4(a) had a lustrous metallic appearance, whereas that corresponding to Fig. 4(c) appeared as a black oxide film.

Figure 5 shows another oxide film site. There were no oxidation products on the grains here, but grinding marks that look like vertical grooves parallel to the circumferential direction were blurred, and multiple microcracks were observed in the axial direction. These cracks were parallel to the cooling channels. During combustion, the inner cylinder surface is compressed because its thermal expansion is constrained by the outer cylinder. Although the metal yields at this time, it becomes tensile during subsequent cooling. It can be assumed that if an oxide film is formed during combustion, cracks due to the tension caused during cooling will occur in it and will remain after exposure to a reducing atmosphere.

Although the metal yields at this time, it becomes tensile during subsequent cooling. It can be assumed that if an oxide film is formed during combustion, cracks will occur in it due to the tension caused during cooling, and that the cracks in the residual oxide film will remain after exposure to a reducing atmosphere.

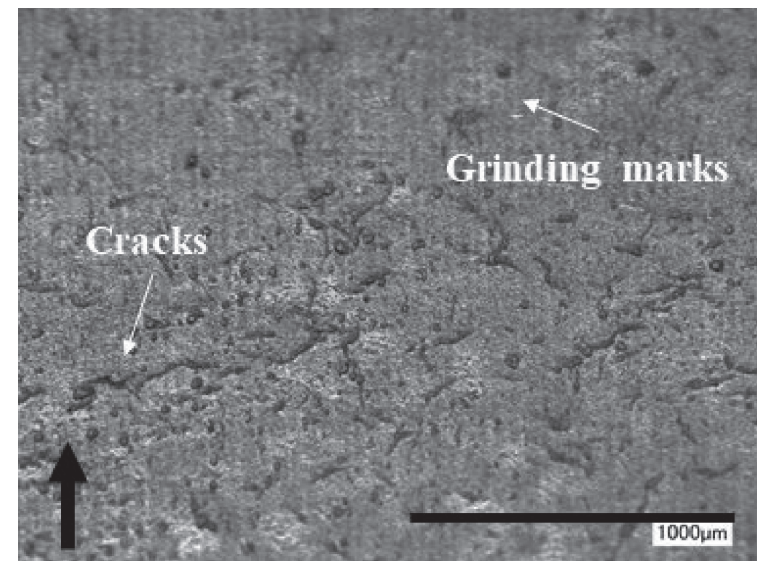

Fig. 5 Microscopic image of a different region of the oxide film with microcracks.

\subsection{Observation of cooling channel deformation near the nozzle throat}

The replica of the nozzle throat showed light and dark streaks parallel to the cooling channel at certain angles of the light source (Fig. 6(a)), with a period of about $3 \mathrm{~mm}$ (Fig. 6(b)). LSM revealed the line profile of its height measurement on a light/dark boundary in Fig. 6(b) (Fig. 6(c)). The dark areas featured multiple cracks in the oxide film that runs parallel to the cooling channel direction,

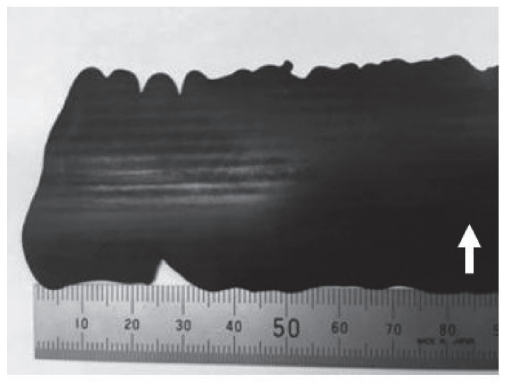

(a)

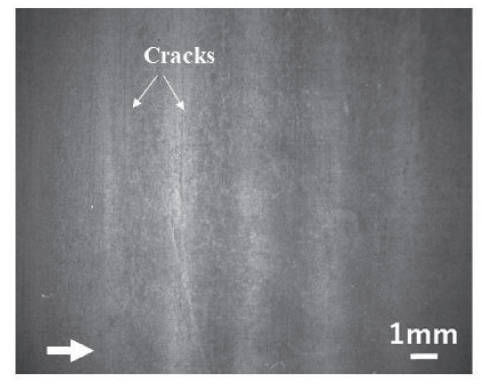

(b)

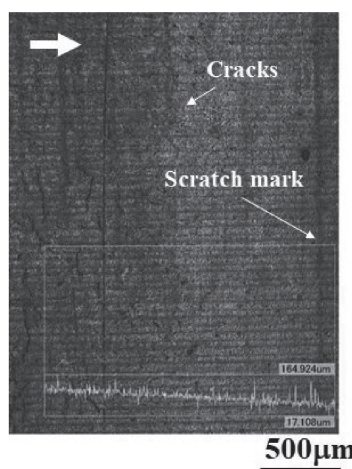

(c)

Fig. 6 Macroscopic images of the sampled replica sheet show light and dark lines parallel to the cooling channels, the macroscopic image (a), the microscopic image (b), laser scanning microscope image of the light and dark line parts and the height measurement profile. 
perpendicular to the grinding traces (Fig. 6(c)). The bright areas, in contrast, had few cracks, and instead featured thick stripes caused by machining on the right side of Fig. 6(b) (Fig. 6(c)). The line profile of the height measurement showed an inclination corresponding to the curvature of the inner cylinder, but no periodic deformation corresponding to the pitch of the cooling channel was observed.

\section{Discussion}

\subsection{Progression of damage in a $\mathrm{Cu}$-alloy combustion chamber}

Molding and visual observation of a $\mathrm{Cu}-\mathrm{Cr}-\mathrm{Zr}$ copper alloy combustion chamber that had undergone 142 combustion cycle tests revealed the following surface properties:

a) Lustrous metal areas (Fig. 4(a)) where grinding marks are clearly visible.

b) Oxide film with oxidation products on grains (Fig. 4(c)), where grinding marks are clearly visible.

c) Oxide film areas with cracks (Fig. 5).

d) Streaky deformed areas parallel to the cooling channels (Fig. 6). Microscopic observation of these areas showed cracks generated on the oxide films.

It appears that the surface damage (Fig. 4(c)) progresses as follows: (1) Thermal stress load and formation of oxide film during combustion; (2) thermal stress load during cooling; and (3) repeated removal of oxide film due to reduction. It follows that the oxide film with oxidation products appears and disappears on the grains (Fig. 4(c)), followed by residual growth of the oxide film, disappearance of grinding marks, and crack formation on the oxide film (Fig. 5).

Considering the mechanism of damage propagation in the combustion chamber on the basis of the deformation behavior during combustion, we can draw the following conclusions: The surface of the inner cylinder is compressed during combustion and, after yielding, becomes tensile during subsequent cooling. Repeated combustion makes the surface uneven owing to the intrusion or extension of sticking slip bands (a typical phenomenon of plastic deformation of metals). If the heat flux during combustion becomes nonuniform as a result of combustion conditions, local nonuniform deformation occurs. Where uneven oxide film generated on the surface during cooling remains, tensile stress acts on it, causing cracking.

\subsection{Methods of evaluating surface damage}

The bright areas on the replica surface parallel to the cooling channels near the nozzle throat (Fig. 6(a), (b)) correspond to the hottest part of the combustion chamber. ${ }^{4}$ We examined creep deformation. The pitch of this light/dark pattern was close to twice the cooling channel pitch $(\sim 1.5 \mathrm{~mm})$. The height line profile shows a slope corresponding to the curvature of the inner cylinder (Fig. 6(c)), but LSM measurements revealed no change in height corresponding to deformation of the cooling channel.

To investigate the cause of this difference in brightness, we examined the surface roughness on the basis of the height measurement results in Fig. 6(c) (Fig. 7). Since the measurement site was near the nozzle throat, a difference in elevation of about $60 \mu \mathrm{m}$ caused by the curvature of the cylinder and a variation of up to $\pm 60 \mu \mathrm{m}$ were observed. Since the inner diameter of the detected part is $\geq 100 \mathrm{~mm}$ and the length of the measurement part is $2 \mathrm{~mm}$, we corrected the inclination for the curvature of the inner wall as:

$$
h_{i}=a P_{i}+b
$$

where $h_{i}$ is the measured value, $P_{i}$ is the distance moved, $a=30.526, b=116.13$, and the error $R^{2}=0.63$. The height data in Fig. 7(a) have considerable variation along the curvature of the inner wall. The reason for the low correlation coefficient is variations in the measurement equipment. The error in the measurement height can be avoided by using a moving average. The most suitable moving average pitch $P_{i}$ is $94 \mu \mathrm{m}$, at which $a=30.116, b=115.85$, and $R^{2}=0.98$. As the moving average value includes the slope and surface roughness, the moving average height $\bar{h}_{i}$, corrected for inclination, can be obtained as:

$$
\bar{h}_{i}=\frac{1}{k+1}\left(h_{i}+\sum_{j=1}^{k} h_{(i+j)}\right)-\left(30.116 P_{i}+115.85\right)
$$

where $k+1=33$ (mean, 94- $\mu$ m steps).

We plotted the results of eq. (2) and light intensity: the height of the unevenness was about $\pm 5 \mu \mathrm{m}$ in the dark zone and about $\pm 3 \mu \mathrm{m}$ in the bright zone (Fig. 8). One possible reason why the light reflection coefficient changes, creating

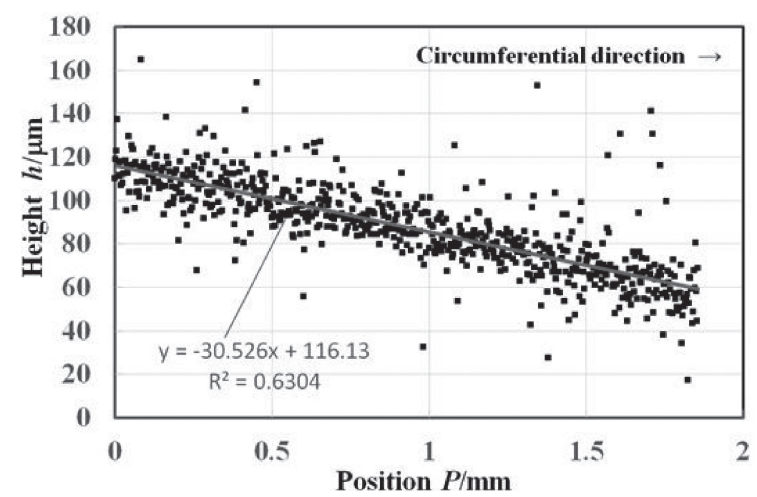

(a)

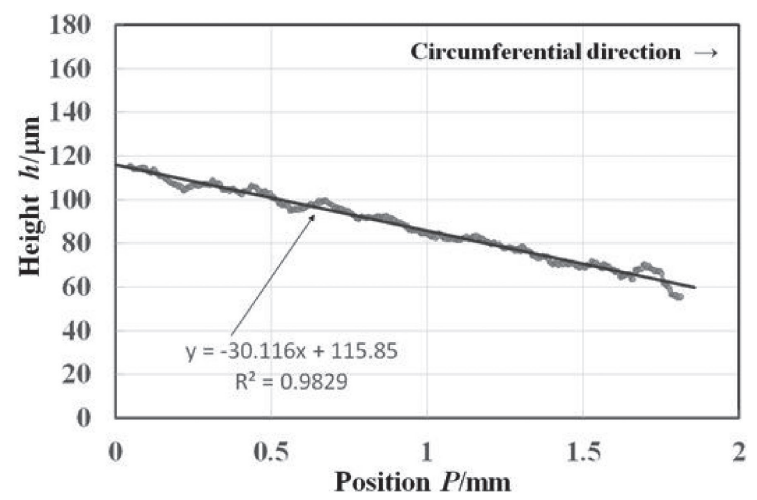

(b)

Fig. 7 Height measurement plots from the laser scanning microscope (a), and with a regression line to estimate inclination correction (b). 


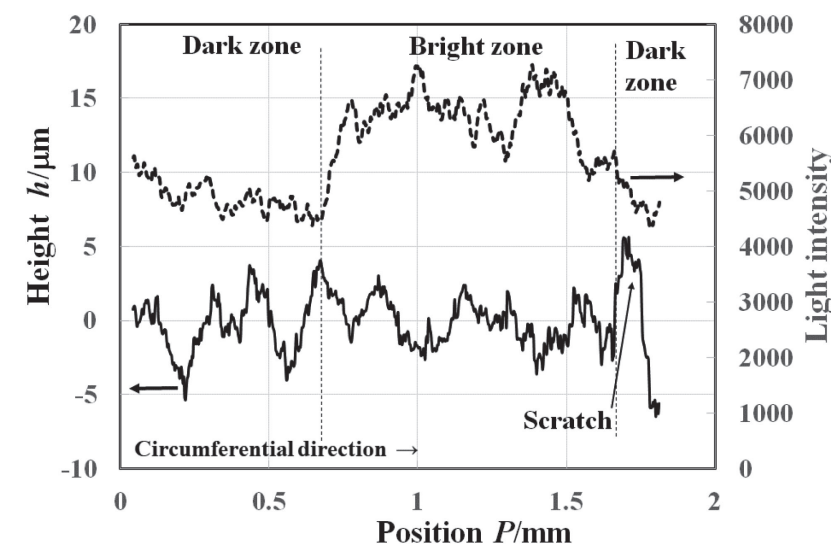

Fig. 8 The light intensity of the laser scanning microscope and the tiltcorrected height.

streaks of light and dark, is that the surface roughness (eq. (2)) increases in relation to the density of cracks generated on the oxide film. One problem when inspecting a crack on an oxide film is knowing whether it is propagating in the surface or in the base material. A study of oxidation and hydrogenation in a $\mathrm{Cu}-\mathrm{Cr}-\mathrm{Zr}$ alloy by small punch testing of its mechanical properties showed the alloy to become very embrittled at $753 \mathrm{~K}$ owing to the effect of internal oxidation. Hydrogen embrittlement was further manifested when this internal oxide was charged with hydrogen. ${ }^{16)}$ Our engine is designed to be operated at up to $700 \mathrm{~K}$, but at higher temperature design points, attention should be paid to the risk of hydrogen embrittlement and internal oxidation.

To be able to accurately evaluate degradation damage to the combustion chamber, it is necessary to fully investigate the cracking behavior of the oxide film during creep fatigue in a redox environment. Further examination of the mechanism of crack growth in the base material is required, taking into account both internal oxidation and hydrogen embrittlement. If cracks in the oxide film progress with cracks in the base material, this replica method, which reveals the characteristics of the oxide film surface without the need for surface treatment of the equipment, is likely to be effective for evaluating degradation damage.

\section{Conclusions}

We examined the potential of the replica method for evaluation of the degradation of a combustion chamber being developed by JAXA.

(1) Although the surface of the chamber's parallel part is curved, replicas could be collected as single $40 \mathrm{~mm} \times$ $180 \mathrm{~mm}$ sheets, showing good transferability of surface properties.

(2) The replicas were able to copy the shapes on the surface of the chamber in all sites: the glossy metallic surface, the oxide film, the continuous traces caused by grinding in the circumferential direction, and scratches in the axial direction that appear to be due to machining.

(3) The mechanism by which surface damage progresses during combustion testing is posited as follows: After the material yields owing to compression during combustion, it becomes tensile during subsequent cooling. Following multiple combustion cycles, irregularities occur on the surface owing to the intrusion and extension of adhering slip bands. If an uneven oxide film generated on the surface remains there during cooling, it is likely to be cracked by tension.

(4) Calculation of the surface roughness while correcting for the inclination of the height measurement result $\bar{h}$ by laser scanning microscopy showed the height of the unevenness to be about $\pm 5 \mu \mathrm{m}$ in dark areas but about $\pm 3 \mu \mathrm{m}$ in light areas. We conclude that the surface roughness increases with greater crack density.

(5) For cracks in the oxide film that follow the base material, our replica method, in which we propose that the value $\bar{h}$ of an oxide film surface that has not been surface-treated be used as the criterion, is potentially effective for evaluating deterioration damage.

\section{REFERENCES}

1) R.T. Cook, E.E. Fryk and J.F. Newell: NASA/CR-168215, (NASA, 1983).

2) M. Murphy, R.E. Anderson, D.C. Rousar and J.A. Van Kleeck: NASA/ CP-2437, (NASA, 1986) pp. 580-616.

3) H. Ogawa, S. Nonaka, Y. Naruo and Y. Inatani: Collected Abstract of The Japan Society for Aeronautical and Space Science Northern Branch, (2015) JSASS-2015-H036.

4) T. Hashimoto, M. Sato, T. Kimura, S. Takada, T. Yagishita, Y. Naruo, H. Ogawa, K. Obase, Y. Fukuda, T. Kai and H. Ohmura: Collected Abstract of The Japan Society for Aeronautical and Space Science Northern Branch, (2015) JSASS-2015-H039.

5) M. Sato, T. Hashimoto, S. Moriya, T. Kimura, T. Yagihashi, T. Masuoka, H. Omura, H. Takagi, T. Fuzii and K. Sato: Symposium on Advanced Materials and Nondestructive Measurements for the Establishment of a Safe and Secure, (JSNDI, 2016) pp. 19-24.

6) M. Shiwa, T. Takatsubo and E. Sato: J. JSNDI 59 (2010) 492-495.

7) E. Sato, H. Tsuda and M. Shiwa: J. JSNDI 62 (2013) 326-327.

8) M. Shiwa and R. Kume: BT Avenue 28, (JAPEIC, 2003) 62-68.

9) S. Mori, K. Tomura and T. Tan: Hitachi Review 69 (1987) 83-88.

10) A.R. Marder: Replication Microscopy Techniques for NDE, (Handbook, Vol. 17: Nondestructive Evaluation and Quality Control, ASM, Russel Township, 1989) pp. 52-56.

11) H. Kashiwaya, Y. Yoshioka, D. Saito and I. Fujiyama: J. JSNDI 46 (1997) 50-57.

12) M. Kamaya: Trans. Jpn. Soc. Mech. Eng. Ser. A 79 (2013) 1530-1544.

13) D. Zuljan and J. Grum: Collected Abstract of The 8th International Conference of the Slovenian Society for Non-Destructive Testing, (Portorož, 2005) pp. 359-368.

14) M. Deguchi, Y. Hori, H. Tobe and E. Sato: J. Jpn. Inst. Copper 54 (2015) 67-72.

15) M. Deguchi, H. Tobe and E. Sato: Int. J. Fatigue 87 (2016) 351-358.

16) S. Komazaki, C. Terada, T. Kutsuzawa and S. Moriya: Trans. Jpn. Soc. Mech. Eng. Ser. A 76 (2010) 1103-1109. 